\title{
Predicting chemosensitivity in osteosarcoma prior to chemotherapy: An investigational study of biomarkers with immunohistochemistry
}

\author{
YONG CHEN $^{1,2}$, YUN YANG $^{3}$, ZHANNA YUAN $^{4}$, CHUNMENG WANG $^{1,2}$ and YINGQIANG SHI ${ }^{1,2}$ \\ ${ }^{1}$ Department of Surgical Oncology, Fudan University Shanghai Cancer Center; ${ }^{2}$ Department of Oncology, Shanghai Medical \\ College, Fudan University, Shanghai 200032; ${ }^{3}$ Department of Bone and Soft Tissue Tumors, Tianjin Medical University \\ Cancer Institute and Hospital; ${ }^{4}$ Key Laboratory of Cancer Prevention and Treatment, Tianjin 300060, P.R. China
}

Received October 14, 2011; Accepted January 23, 2012

DOI: $10.3892 / \mathrm{ol} .2012 .604$

\begin{abstract}
Osteosarcoma has one of the worst prognoses in adolescents; only $20-60 \%$ of patients have high rates of histological necrosis with intensive neoadjuvant chemotherapy. In this study, we investigated the prognostic values of hypoxia-inducible factor $1 \alpha$ (HIF-1 $\alpha$ ), apurinic endonuclease 1 (APE1), vascular endothelial growth factor (VEGF) and cycloogenase-2 (COX-2) protein expression and their predictive value of tumor necrosis rate and prognosis, as well as their interrelationships. Formalin-fixed paraffin-embedded tissue samples were obtained from 49 patients with osteosarcoma. Immunohistochemistry assays were performed in pre-chemotherapy samples to determine HIF-1 $\alpha$, VEGF, APE1 and COX-2 protein expression levels and hematoxylin and eosin staining was performed in post-operative samples to determine the tumor necrosis rate. Univariate and multivariate analyses were used to assess the impact of protein expression on prognosis. HIF-1 $\alpha$ was significantly correlated with every protein we tested: VEGF $(\mathrm{P}=0.032)$, APE1 $(\mathrm{P}<0.001)$ and COX-2 $(\mathrm{P}<0.001)$. HIF-1 $\alpha$ protein expression had a significant impact on disease-free survival $(\mathrm{P}=0.006)$. Expression of HIF-1 $\alpha$ had a sensitivity of $64.7 \%$ and a specificity of $71.9 \%$ for a poor pathological response $(<90 \%$ tumor necrosis) versus a good pathological response ( $\geq 90 \%$ tumor necrosis). In conclusion, expression of HIF- $1 \alpha$ is a predictor of tumor response to neoadjuvant chemotherapy and outcome in osteosarcoma, and correlates with VEGF, APE1 and COX-2.
\end{abstract}

Correspondence to: Dr Yong Chen, Department of Surgical Oncology, Fudan University Shanghai Cancer Center, 270 Dongan Road, Xuhui, Shanghai 200032, P.R. China

E-mail: chenyong780417@gmail.com

Key words: hypoxia-inducible factor $1 \alpha$, apurinic endonuclease-1, vascular endothelial growth factor, cycloogenase-2, osteosarcoma

\section{Introduction}

Osteosarcoma (OS) is the most common malignant bone tumor in adolescents and young adults. The use of neoadjuvant chemotherapy and improvements in surgical technology have increased the survival rate to $65-75 \%$ (1). However, pulmonary metastasis occurs in approximately $40 \%$ of patients and remains the major cause of mortality. With intensive preoperative chemotherapy, high rates of histological necrosis $(\geq 90 \%)$, and a strong prognostic factor predicting a favorable outcome, have been reported in only $20-60 \%$ of patients $(2,3)$, making it necessary to identify new markers or gene sets that would predict tumor response to chemotherapy, and allow more individually adapted multimodality treatments.

Several candidate markers for chemosensitivity and prognosis have been identified. Hypoxia-inducible factor $1 \alpha$ (HIF-1 $\alpha$ ) has been demonstrated to correlate with an unfavorable prognosis in a number of types of cancer, and is known to be significant in chemoresistance (4-6). Findings of recent studies have shown a significant association between the expression of HIF-1 $\alpha$ and prognosis in OS $(7,8)$, inhibition of HIF-1 $\alpha$ gene expression by siRNA decreased tumor formation rate and growth speed in xenograft mice (9). Results of previous studies on human OS cells demonstrated that hypoxia-induced overexpression of HIF-1 $\alpha$ was associated with the upregulation of the vascular endothelial growth factor (VEGF) and tumor angiogenesis (10). VEGF is not only a key factor in the angiogenesis pathway, but also a target for several therapeutics approved by the U.S. Food and Drug Administration (11). Apurinic endonuclease 1 (APE1) has been discussed as a potential drug target in various cancer types, as its expression has been linked to chemosensitivity and prognosis $(12,13)$. In OS, a previous study indicated that APE1 expression was an independent predictor of OS local recurrence and/or metastasis (13). The respect in which HIF-1 $\alpha$ and APE1 are coexpressed in OS and the impact of their coexpression on the tumor necrosis rate requires further investigation. The expression of cycloogenase-2 (COX-2) is known to be markedly correlated with clinical stage and prognosis in patients with OS (14). Inhibition of COX-2 is capable of inducing massive tumor necrosis in xenograft mice (15). A previous 
study revealed a correlation between COX-2 and VEGF in breast ductal carcinoma (16). As with APE1 and HIF-1 $\alpha$, the correlation has not been sufficiently examined in OS.

In this study, we investigated the prognostic values of HIF-1 $\alpha$, APE1, VEGF and COX-2 protein expression as well as their interrelationships in OS. We further analyzed the correlation of these proteins with clinical and histopathological variables, including tumor size, primary American Joint Committee on Cancer (AJCC) stage (17), metastasis, survival, and particularly tumor necrosis rate following neoadjuvant chemotherapy.

\section{Materials and methods}

Patients and treatment protocols. A total of 49 consecutive cases (28 males and 21 females; median age, 18.5 years; age range, 11-72 years) with non-metastatic primary OS were selected for the study. The patients were treated in the Department of Bone and Soft Tissue Tumors at the Tianjin Medical University Cancer Institute and Hospital between 2000 and 2009. All the patients underwent incisional biopsy for diagnosis prior to neoadjuvant chemotherapy and we used the remaining tissue for immunohistochemistry. The neoadjuvant chemotherapy agents used were in accordance with those proposed and proven previously, including methotrexate (MTX), adriamycin (ADM), cisplatin (CDDP) and ifosfamide (IFO) (18). In brief, patients were administered $10 \mathrm{~g} / \mathrm{m}^{2}$ of MTX in week 1 and $12 \mathrm{~g} / \mathrm{m}^{2}$ of IFO in week 2 , followed by $80 \mathrm{mg} / \mathrm{m}^{2}$ of CDDP and $60 \mathrm{mg} / \mathrm{m}^{2}$ of combined ADM in week 3 . Following two weeks of rest, lesions were reassessed using computed tomography or magnetic resonance imaging studies. The pre- and post-chemotherapy imaging data were reviewed by radiologists who were blind to the clinical data, and reassessed by surgical oncologists for resectability and change in scope.

Further treatments were performed according to the reassessments. Patients expected to have a safe margin and acceptable function outcome were assigned to surgery and post-operative adjuvant chemotherapy. Patients with irresectable diseases or those expected to have an unacceptable function impairment were assigned to amputation when the disease progressed. Patients with a tumor response to neoadjuvant chemotherapy, but no decrease in scope of surgery were treated with second cycle neoadjuvant chemotherapy followed by surgical resection. Tumor size data were available based on a review of the pre-treatment imaging studies and the pathology reports. We assessed chemotherapy efficacy histologically using the Huvos grading system post-operatively (19). According to the percentage of dead cells, the 49 cases were divided into two groups; tumors demonstrating a good response to preoperative chemotherapy (dead cells $\geq 90 \%$, surviving cells $\leq 10 \%$ ) and tumors demonstrating a poor response (surviving cells $>10 \%$ ). Post-operative chemotherapy was prescribed according to the tumor necrosis rate. Patients with a good response were treated with the same dosage of therapeutic agents as administered pre-operatively. Patients with a poor response were treated with intensified doses (MTX, $12 \mathrm{~g} / \mathrm{m}^{2}$; IFO, $15 \mathrm{~g} / \mathrm{m}^{2}$; CDDP, $100 \mathrm{mg} / \mathrm{m}^{2}$ and ADM, $80 \mathrm{mg} / \mathrm{m}^{2}$ ). The mean follow-up time was 29 months (range, 6-100). Disease-free survival (DFS) was defined from the day of surgery until the
Table I. Patient characteristics $(n=49)$.

\begin{tabular}{lc}
\hline Parameters & $\mathrm{n}$ \\
\hline Total & $49(100 \%)$ \\
Age, median (range) & $18.5(11-72)$ \\
Gender & \\
Male & $28(57 \%)$ \\
Female & $21(43 \%)$ \\
AJCC ${ }^{\mathrm{a}}$ stage & \\
IIA & $11(22 \%)$ \\
IIB/III & $38(78 \%)$ \\
Size, cm & \\
Median (range) & \\
Neoadjuvant chemotherapy cycles & $(3.5-15.0)$ \\
1 cycle & $24(49)$ \\
2 cycles & $25(51)$ \\
Surgery & \\
Limb-salvage & $44(90)$ \\
Amputation & $5(10)$ \\
Follow-up (mean) & 29 \\
\hline
\end{tabular}

${ }^{\mathrm{a} A m e r i c a n}$ Joint Committee on Cancer.

first relapse of the disease or mortality. Mortality from a cause other than OS, or survival until the end of the observation period, was considered a censoring event.

This study was approved by Fudan University Cancer Center and Shanghai Medical College, as well as Tianjin Medical University Cancer Institute and Hospital and Tianjin Key Laboratory of Cancer Prevention and Treatment. Patient consent was obtained prior to obtaining specimens and reviewing patient information.

Immunohistochemistry. Formalin-fixed, paraffin-embedded tissues obtained in the biopsy procedures were cut consecutively into 3- $\mu \mathrm{m}$ sections. The histological slides were deparaffinized in xylol. The slides were heated in $0.01 \mathrm{M}$ citrate buffer for $10 \mathrm{~min}$ in a microwave oven. Following $20 \mathrm{~min}$ of cooling and washing in PBS, endogenous peroxidase was blocked with methanol containing $0.3 \%$ hydrogen peroxide for $30 \mathrm{~min}$, followed by incubation with PBS for $30 \mathrm{~min}$. For the immunohistochemical detection of HIF-1 $\alpha$, specimens were incubated overnight at $4^{\circ} \mathrm{C}$ with the primary antibodies; HIF-1 $\alpha(1: 100$, Santa Cruz Biotechnology Inc., Santa Cruz, CA, USA), APE1 (1:100, Abcam, Cambridge, UK), VEGF (1:200, Santa Cruz Biotechnology Inc.) and COX-2 (1:100, Abcam). The quality (number, intensity and pattern) of every staining procedure for the proteins was comparatively evaluated using the consecutive control sections. The tumor cell immunoreactivity of HIF-1 $\alpha$, APE1 and COX-2 were separated into four groups $(7,13,14)$ : negative, mild positive, moderate positive and strong positive. The tumor cell immunoreactivity of VEGF was separated into two groups as reported previously (8): negative and positive. The assessments were performed independently by two experienced investigators blinded to the patient clinicopathological data. 
Table II. Correlation between clinicopathological parameters and HIF-1 $\alpha$ protein expression in osteosarcoma $(\mathrm{n}=49)$.

\begin{tabular}{|c|c|c|c|c|c|c|}
\hline \multirow[t]{2}{*}{ Parameters } & \multicolumn{4}{|c|}{ HIF-1 $\alpha$} & \multirow[b]{2}{*}{$\mathrm{r}_{\mathrm{s}}$} & \multirow[b]{2}{*}{ P-value } \\
\hline & - & $1+$ & $2++$ & $3+++$ & & \\
\hline $\operatorname{Age}^{\mathrm{a}}$ & \multicolumn{4}{|c|}{$18.5(11-72)$} & 1.584 & 0.663 \\
\hline Tumor size $(\mathrm{cm})^{\mathrm{b}}$ & $8.28 \pm 2.53$ & $9.91 \pm 2.73$ & $11.59 \pm 0.93$ & $12.75 \pm 1.20$ & 10.189 & $<0.001$ \\
\hline pTNM stage & & & & & 0.310 & 0.030 \\
\hline IIA & 7 & 4 & 0 & 0 & & \\
\hline IIB/III & 15 & 6 & 9 & 8 & & \\
\hline Metastasis & & & & & 0.380 & 0.007 \\
\hline No & 18 & 5 & 4 & 3 & & \\
\hline Yes & 4 & 5 & 5 & 5 & & \\
\hline Tumor necrosis rate & & & & & -0.464 & 0.001 \\
\hline$<90 \%$ & 3 & 6 & 6 & 5 & & \\
\hline$\geq 90 \%$ & 19 & 4 & 3 & 3 & & \\
\hline
\end{tabular}

P-values were calculated by Spearman's rank correlation test $(\mathrm{n}=49)$; ${ }^{\mathrm{E}}$ Expressed as median (range), mean ${ }^{2}=1.584, \mathrm{P}=0.663$ (Kruskal-Wallis test); ${ }^{b}$ Expressed as mean, $\mathrm{F}=10.189, \mathrm{P}<0.001$ (ANOVA test). HIF-1 $\alpha$, hypoxia-inducible factor $1 \alpha$.

Table III. Multivariate analysis of factors associated with prognosis in osteosarcoma $(n=49)$.

\begin{tabular}{llll}
\hline & & \multicolumn{2}{c}{ Disease-free survival } \\
\cline { 2 - 5 } Factors & Risk ratio & 95\% confidence interval & \\
\hline pTNM stage III/II & 2.339 & $0.232-23.578$ & 0.019 \\
Tumor necrosis rate $\geq 90 \% / \leq 90 \%$ & 0.123 & $0.023-0.663$ & 0.015 \\
HIF-1 $\alpha$ positive/negative & 6.068 & $1.107-33.257$ & 0.038 \\
APE1 positive/negative & 9.728 & $1.408-67.190$ & 0.021
\end{tabular}

HIF-1 $\alpha$, hypoxia-inducible factor $1 \alpha$; APE1, apurinic endonuclease 1.

Statistical analysis. SPSS 13.0 statistical software was used for statistical analysis. The correlation between the protein expression levels and the clinicopathological parameters were tested using the Spearman's test for bivariate correlations. The variables considered for their prognostic value included age at diagnosis, gender, tumor size, margin status, chemotherapy, tumor necrosis rate and protein expression. Survival curves were computed by the Kaplan-Meier method and compared by the log-rank test. Multivariate analyses based on the stepwise Cox proportional hazards model were used to identify the most significant factors related to outcome. A stepwise forward selection procedure was used, and a significance level of 5\% was selected as the criterion for entering factors in the multivariate model. The association between HIF-1 $\alpha$ expression and various clinicopathological characteristics were analyzed using the Chi-square test. $\mathrm{P}<0.05$ was considered to indicate a stastically significant difference.

\section{Results}

Correlation of HIF-1 $\alpha$ protein expression with clinicopathological parameters and tumor necrosis rate. The clinicopathological characteristics of the patients are shown in Table I. Amputation was performed in 5 patients and the limb-salvage rate was $90 \%(44 / 49)$ in all patients. Of the 49 patients, HIF-1 $\alpha$ was negative in 22 patients (44.9\%), mild positive in $10(20.4 \%)$, moderate positive in $9(18.4 \%)$ and strong positive in 8 (16.3\%). The correlation between HIF-1 $\alpha$ expression and the clinicopathological parameters is shown in Table II. HIF-1 $\alpha$ expression was significantly associated with tumor size $(\mathrm{P}<0.001)$, AJCC stage $(\mathrm{P}=0.030)$, metastasis $(\mathrm{P}=0.007)$ and tumor necrosis rate $(\mathrm{P}=0.001)$. Other clinicopathological parameters, including gender, age and surgery type were not associated with HIF-1 $\alpha$ expression.

HIF-1 $\alpha$ expression was correlated to prognostic factors APE1, $V E G F$ and COX-2 in patients with osteosarcoma. We firstly observed that HIF-1 $\alpha$ expression was associated with DFS using a univariate analysis (Fig. 1A), and an independent prognosticator in OS ( $\mathrm{P}=0.038)$ (Table III). APE1 was recognized as a co-factor that modulates the expression of HIF-1 $\alpha$. We then investigated whether APE1 expression was prognostic in OS and its correlation with HIF-1 $\alpha$. Of the 49 patients, the APE1 protein was negative in 17 patients (34.7\%), mild positive in 13 
A

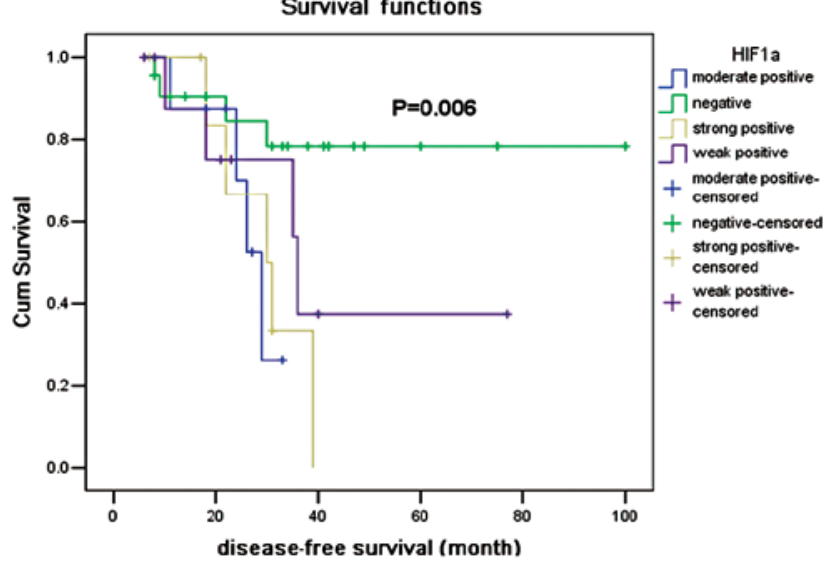

C

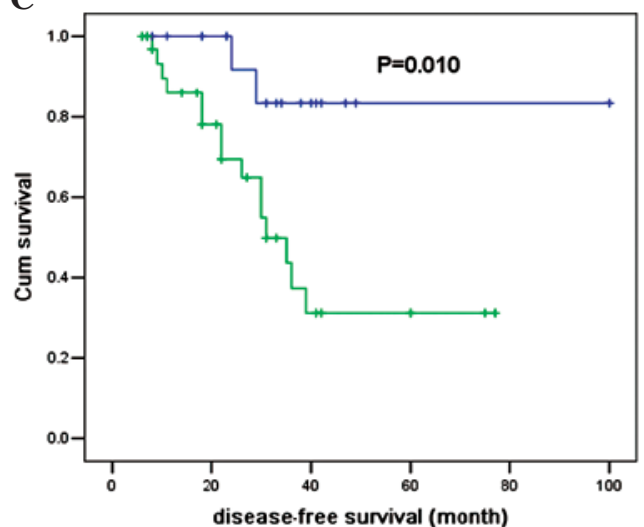

$\mathbf{E}$

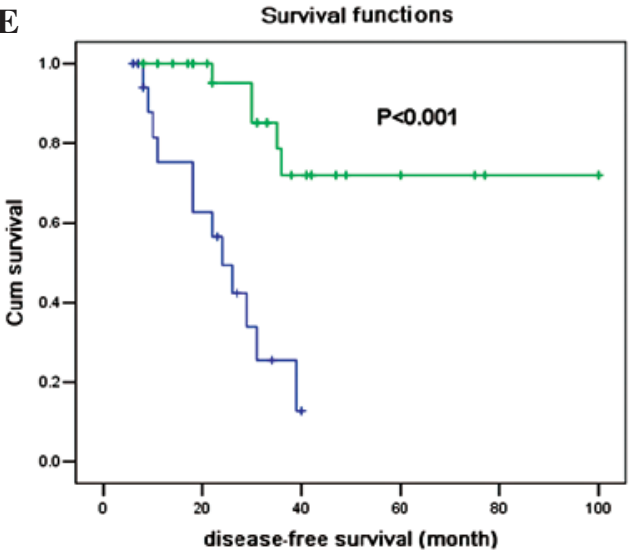

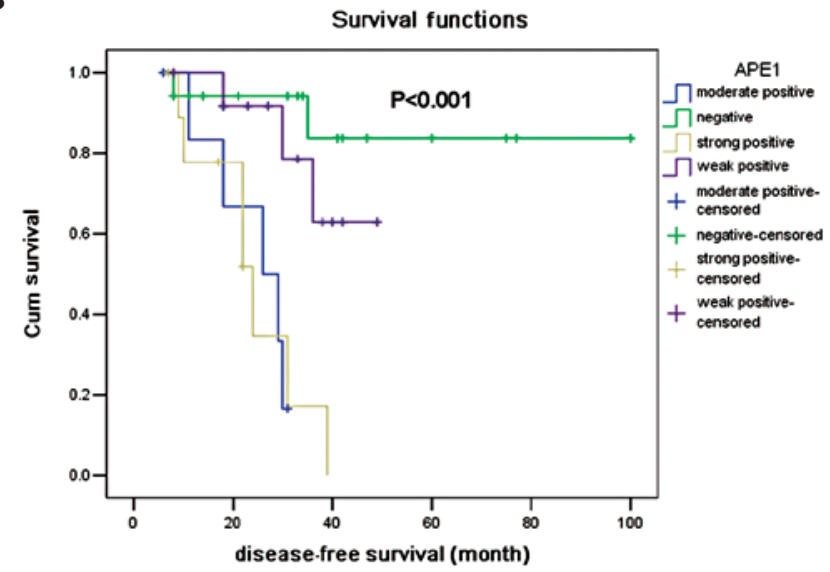

D

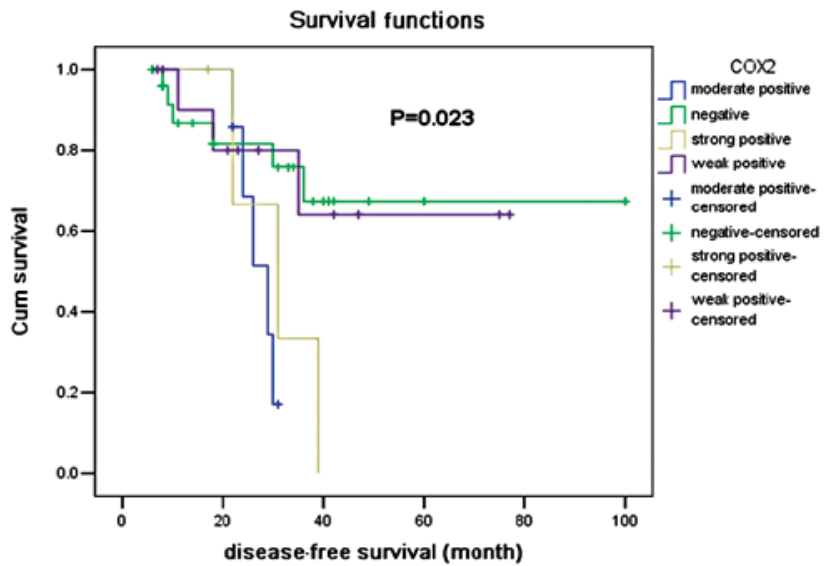

$\mathbf{F}$

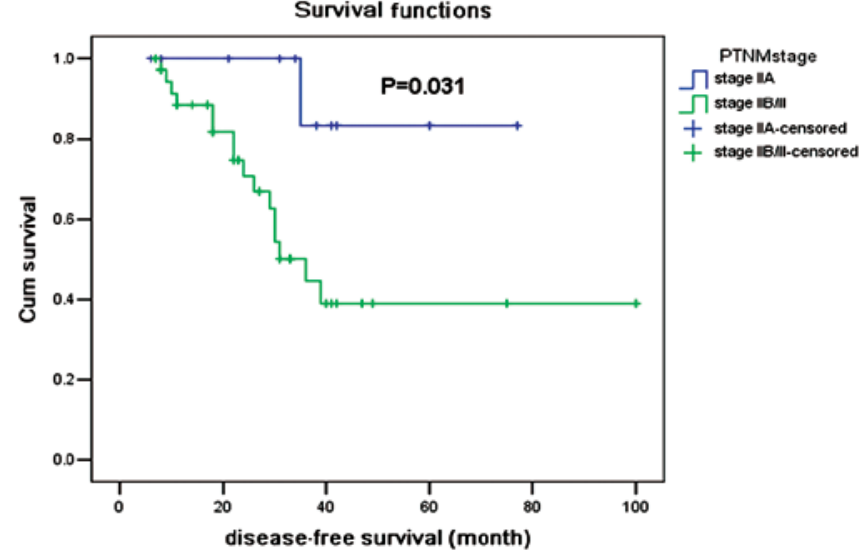

Figure 1. Curves of disease free survival according to (A) HIF-1 $\alpha$ expression; (B) APE1 expression; (C) VEGF expression; (D) COX-2 expression; (E) Tumor necrosis rate and (F) AJCC stage. HIF-1 $\alpha$, hypoxia-inducible factor $1 \alpha$; APE1, apurinic endonuclease 1; VEGF, vascular endothelial growth factor; COX-2, cycloogenase-2.

$(26.5 \%)$, moderate positive in $8(16.3 \%)$ and strong positive in 11 patients $(22.5 \%)$. APE1 protein expression was significantly associated with DFS ( $\mathrm{P}<0.001)$ (Fig. 1B). Expression of APE1 significantly correlated with HIF-1 $\alpha$ expression $(\mathrm{P}<0.001)$ and tumor necrosis rate $(\mathrm{P}<0.001)$.

VEGF was accepted to be a target of HIF-1 $\alpha$ and its expression was reported to be associated with tumor growth and metastasis. A previous study has demonstrated that in OS patients the survival rate of those with VEGF expression was substantially worse than patients without VEGF expression (20). Of the 49 patients, the VEGF protein was negative in 17 patients $(34.7 \%)$ and positive in $32(65.3 \%)$.
The VEGF protein expression was confirmed to be predictive for DFS (Fig. 1C) $(\mathrm{P}=0.010)$. The expression of VEGF was significantly correlated with HIF-1 $\alpha$ expression $(\mathrm{P}=0.032)$. This correlation is in accordance with a previous study in OS cell lines (21). VEGF expression was also corrrelated with APE1 $(\mathrm{P}=0.014)$.

COX-2 is a significant mediator in tumor invasion and metastasis, and has been investigated to be a target for cancer therapy (14). In our study, COX-2 protein was negative in 25 patients $(51.0 \%)$, mild positive in $12(24.5 \%)$, moderate positive in $7(14.3 \%)$ and strong positive in $5(10.2 \%)$. COX-2 expression was also associated with DFS ( $\mathrm{P}=0.023)$ (Fig. 1D). 
As shown in Table II, expression of COX-2 was significantly correlated with HIF-1 $(\mathrm{P}<0.001)$, APE1 $(\mathrm{P}<0.001)$ and VEGF $(\mathrm{P}=0.033)$.

Sensitivity and specificity in predicting tumor necrosis rate. We aimed to find a marker to predict the tumor necrosis rate prior to adjuvant chemotherapy in OS patients. As a single predictor, a high expression of HIF-1 $\alpha$ and/or APE1 was significantly associated with poor tumor necrosis rate. A total of $71.9 \%(23 / 32)$ of patients with a negative/mild positive HIF-1 $\alpha$ expression had a good pathological response to neoadjuvant chemotherapy, and $80.0 \%(24 / 30)$ of patients with a negative/mild positive APE1 expression had a tumor necrosis rate of no less than $90 \%$. By contrast, $64.7 \%$ (11/17) of patients with moderate/ strong positive HIF-1 $\alpha$ expression revealed a poor response to neoadjuvant chemotherapy, while $73.7 \%$ (14/19) of patients with a moderate/strong positive APE1 expression had a tumor necrosis rate of $<90 \%$. In patients with a negative/mild positive expression of HIF- $1 \alpha$ and APE1, 88.5\% (23/26) of patients had a good pathological response to neoadjuvant chemotherapy, while in those with a moderate/strong positive expression of HIF- $1 \alpha$ and APE1, 75.0\% (9/12) had a tumor necrosis rate of $<90 \%$, indicating that the expression of HIF- $1 \alpha$ and APE1 is a potential marker in predicting tumor responses to chemotherapy in OS.

\section{Discussion}

In this study, we determined the protein expression of HIF-1 $\alpha$, VEGF, APE1 and COX-2 in the pre-chemotherapy biopsy samples of OS patients and determined the tumor necrosis rate in their post-chemotherapy tumors. By using immunohistochemistry analysis, we hoped to achieve a more precise understanding of the associations of these protein expressions with each other and with patients' prognosis. Using the multivariate Cox regression analysis, it was revealed that HIF-1 $\alpha$ and APE1 protein expression demonstrated the most significant impact on DFS in our patient cohort $(\mathrm{P}=0.009)$.

The tumor necrosis rate following pre-operative chemotherapy (histological response) is generally accepted to be the strongest prognostic factor for OS patients without metastases at initial diagnosis and it is also an indicator for post-operative chemotherapy (22). Our study also demonstrated that the tumor necrosis rate (Fig. 1E) and the AJCC stage (Fig. 1F) were prognostic factors in OS. Recently, increasing efforts have emerged to find accessible markers to predict tumor response prior to administration of neoadjuvant chemotherapy in OS, but the significance of RANKL expression in predicting a pathological response was concluded from a small cohort study, in which the pathological response to pre-operative chemotherapy was not correlated with survival (23). Other markers, including plasma proteome and microRNAs, require invasive procedures and are unable to present the intrinsic characteristics of the tumor samples or provide potential targets for improvements prior to neoadjuvant chemotherapy $(24,25)$. HIF-1 $\alpha$ overexpression was proven to be associated with a decreased response to chemotherapy in various cancer types $(4,5)$, but little data were available which correlated HIF-1 $\alpha$ and APE1 expression with the tumor necrosis rate in OS. In this study, we determined that HIF-1 $\alpha$ and APE1 expression may be reliable predictors of pathological responses to neoadjuvant chemotherapy in OS patients, indicating that a positive expression of HIF-1 $\alpha$ and APE1 may be new markers for selecting patients in whom neoadjuvant chemotherapy is not enough and a combinational targeted therapy is required.

The results of this pilot study also demonstrate a strong correlation between HIF-1 $\alpha$ and VEGF, which emphasizes the role of HIF-1 $\alpha$ in angiogenesis and its most prominent markers VEGF and OS. Although we did not find a significant correlation between VEGF expression and the tumor necrosis rate, our results showed that a high VEGF expression significantly correlated with poorer survival $(\mathrm{P}=0.010)$.

COX-2 expression has recently been correlated with $\mathrm{HIF}-1 \alpha$ and VEGF. As a novel target, COX-2 has been investigated for cancer therapy. In our study, COX-2 is significantly associated with metastasis, indicating that patients, particularly with a positive expression of COX-2, may require anti-COX-2 treatment in adjuvant therapies to prevent tumor metastasis and improve long term metastasis-free survival.

In conclusion, our study has provided supporting evidence that HIF-1 $\alpha$ protein expression is associated with the pathological response and outcome in OS patients. This expression correlated with upstream APE1 and downstream VEGF and COX-2. The protein expression of HIF-1 $\alpha$ and APE1 may be a potential marker and predict pathological response to neoadjuvant chemotherapy. More studies are required to investigate whether their inhibitors are capable of increasing chemosensitivity and improving prognosis in osteosarcoma patients, particularly those with poor pathological responses to neoadjuvant chemotherapy.

\section{Acknowledgements}

This project was financially supported by the National Natural Science Foundation of China (Grant Number 30900596). The authors thank Dr FengJu Song from the Department of Epidemiology in the Key Laboratory of Cancer Prevention and Treatment in Tianjin for statistical assistance.

\section{References}

1. Mankin HJ, Hornicek FJ, Rosenberg AE, Harmon DC and Gebhardt MC: Survival data for 648 patients with osteosarcoma treated at one institution. Clin Orthop Relat Res 429: 286-291, 2004.

2. Bramwell VHC: The role of chemotherapy in osteogenic sarcoma. Crit Rev Oncol Hematol 20: 61-85, 1995.

3. Bramwell VHC: The role of chemotherapy in the management of non-metastatic operable extremity osteosarcoma. Semin Oncol 24: 561-571, 1997.

4. Liu L, Ning X, Sun L, Zhang H, Shi Y, Guo C, et al: Hypoxia-inducible factor-1 alpha contributes to hypoxia-induced chemoresistance in gastric cancer. Cancer Sci 99: 121-128, 2008.

5. Quintero M, Mackenzie N and Brennan PA: Hypoxia-inducible factor $1(\mathrm{HIF}-1 \alpha)$ in cancer. Eur J Surg Oncol 30: 465-468, 2004.

6. Russell DL, Ian S and Adrian L: The role of hypoxia-inducible factor-1 in three dimensional tumor growth, apoptosis and regulation by the insulin-signaling pathway. Cancer Res 65: 4147-4152, 2005.

7. Yang QC, Zeng BF, Dong Y, Shi ZM, Jiang ZM and Huang J: Overexpression of hypoxia-inducible factor-1a in human osteosarcoma: correlation with clinicopathological parameters and survival outcome. Jpn J Clin Oncol 37: 127-134, 2007.

8. Mizobuchi H, Castellano JMG, Philip S, Healey JH and Gorlick R: Hypoxia markers in human osteosarcoma: an exploratory study. Clin Orthop Relat Res 466: 2052-2059, 2008. 
9. Wu Q, Yang SH, Ye SN and Wang RY: Therapeutic effects of RNA interference targeting HIF-1 alpha gene on human osteosarcoma. Nail Med J Chin 85: 409-413, 2005.

10. Cai W, Chen A, Guo F and Zhu B: Effects of HIF-1 $\alpha$ antisense oligonucleotides on the expression of HIF-1 $\alpha$ and VEGF in the osteosarcoma cell line MG-63. Chi J Clin Oncol 33: 1-5, 2006.

11. Ho QT and Kuo CJ: Vascular endothelial growth factor: biology and therapeutic applications. Int J Biochem Cell Biol 39: 1349-1357, 2007.

12. Sak SC, Harnden P, Johnston CF, Paul AB and Kiltie AE: APE1 and XRCC1 protein expression levels predict cancer-specific survival following radical radiotherapy in bladder cancer. Clin Cancer Res 11: 6205-6211, 2005.

13. Yang JL, Yang D, Cogdell D, Du XL, Li HX, Pang Y, et al: APEX1 gene amplification and its protein overexpression in osteosarcoma: correlation with recurrence, metastasis and survival. Technol Cancer Res Treat 9: 161-169, 2010.

14. Geng YH, Wan CX and Chen PH: Expressions of Cox-2 and HIF-l $\alpha$ and their relationship with clinicopathologic characteristics of osteosarcoma. Tumor 28: 427-430, 2008.

15. Dickens S and Cripe TP: Effect of combined cyclooxygenase-2 and matrix metalloproteinase inhibition on human sarcoma xenografts. J Pediatr Hematol Oncol 25: 709-714, 2003.

16. Subbaramaiah K and Dannenberg AJ: Cyclooxygenase 2: a molecular target for cancer prevention and treatment. Trends Pharmacol Sci 24: 96-102, 2003.

17. American Joint Committee on Cancer: AJCC Cancer Staging Manual. Greene FL, Balch CM, Page DL, Haller DG, Fleming ID Morrow M and Fritz AG (eds). 6th edition. Springer-Verlag, New York, 2002.
18. Ferrari S and Palmerini E: Adjuvant and neoadjuvant combination chemotherapy for osteogenic sarcoma. Curr Opin Oncol 19: 341-346, 2007.

19. Rosen G, Caparros B, Huvos AG, et al: Preoperative chemotherapy for osteogenic sarcoma: selection of post-operative adjuvant chemotherapy based on the response of the primary tumor to preoperative chemotherapy. Cancer 49: 1221-1230, 1982.

20. Kaya M, Wada T, Akatsuka T, et al: Vascular endothelial growth factor expression in untreated osteosarcoma is predictive of pulmonary metastasis and poor prognosis. Clin Cancer Res 6: 572-577, 2000.

21. Wu Q, Yang SH, Wang RY, Ye SN, Xia T and Ma DZ: Effect of silencing HIF-1 $\alpha$ by RNA interference on expression of vascular endothelial growth factor in osteosarcoma cell line SaOS-2 under hypoxia. Chin J Cancer 24: 531-535, 2005.

22. Bielack SS, Kempf-Bielack B, Delling G, et al: Prognostic factors in high-grade osteosarcoma of the extremities or trunk: an analysis of 1,702 patients treated on neoadjuvant cooperative osteosarcoma study group protocols. J Clin Oncol 20: 776-790, 2002.

23. Lee JA, Jung JS, Kim DH, et al: RANKL expression is related to treatment outcome of patients with localized, high-grade osteosarcoma. Pediatr Blood Cancer 56: 738-743, 2011.

24. Li Y, Dang TA, Shen J, Hicks J, Chintagumpala M, Lau CC, et al: Plasma proteome predicts chemotherapy response in osteosarcoma patients. Oncol Rep 25: 303-314, 2011.

25. Gougelet A, Pissaloux D, Besse A, Perez J, Duc A, Dutour A, et al: Micro-RNA profiles in osteosarcoma as a predictive tool for ifosfamide response. Int J Cancer 129: 680-690, 2011. 